\title{
Self-propulsion of active droplets without liquid-crystalline order
}

\author{
Rajesh Singh $\odot,{ }^{1, *}$ Elsen Tjhung, ${ }^{1,2}$ and Michael E. Cates ${ }^{1}$ \\ ${ }^{1}$ DAMTP, Centre for Mathematical Sciences, University of Cambridge, Wilberforce Road, Cambridge CB3 OWA, United Kingdom \\ ${ }^{2}$ Department of Physics, University of Durham, Science Laboratories, South Road, Durham DH1 3LE, United Kingdom
}

(Received 13 April 2020; accepted 13 July 2020; published 23 July 2020)

\begin{abstract}
The swimming of cells, far from any boundary, can arise in the absence of long-range liquid-crystalline order within the cytoplasm, but simple models of this effect are lacking. Here, we present a two-dimensional model of droplet self-propulsion involving two scalar fields, representing the cytoplasm and a contractile cortex. An active stress results from coupling between these fields; self-propulsion results when rotational symmetry is spontaneously broken. The swimming speed is predicted, and shown numerically, to vary linearly with the activity parameter and with the droplet area fraction. The model exhibits a Crowley-like instability for an array of active droplets.
\end{abstract}

DOI: 10.1103/PhysRevResearch.2.032024

Active fluids are an emerging class of nonequilibrium systems, where energy is injected into the system locally and continuously, by the constituent particles themselves [1]. Many examples of active fluids are biological in nature, for example, actomyosin networks inside the cell cytoskeleton [2-4] and dense suspensions of microtubules and kinesins in vitro [5,6]. In the case of actomyosin networks, each myosin motor can attach (and detach) to two actin filaments and pull the two filaments inwards, causing a net local contractile stress, which drives the system out of equilibrium. In many cases, this local energy injection at the filament scale can be translated into a macroscopic motion. For example, actomyosin contraction at the rear of the cell cortex has been shown to play an important role in the swimming motility of cells in a bulk fluid environment [3,4], far from any boundary at which crawling can instead occur.

At the level of phase-field modeling and simulations, cell motility is often described as the spontaneous motion of a droplet of active fluid [7-10]. The current field-theoretic understanding of cell swimming involves a scalar field $\phi$, coupled to polar or nematic liquid-crystalline order, described by a vector or a second-rank tensor $[7,8,11]$. The scalar field delineates the cell's interior $(\phi>0)$ from its exterior $(\phi<0)$ whereas the vectorial/tensorial field describes bulk internal alignment of a uniform or cortical "cytoskeleton." The propulsion mechanism then relies on a discrete broken symmetry along a preexisting axis of orientational order, such as a spontaneous splay transition [11], or self-advection caused by net polymerization at the leading end of each polar filament $[7,10,12]$. Thus, the vectorial/tensorial nature of the order parameter is crucial to obtain self-propulsion in such

\footnotetext{
*rs2004@ cam.ac.uk

Published by the American Physical Society under the terms of the Creative Commons Attribution 4.0 International license. Further distribution of this work must maintain attribution to the author(s) and the published article's title, journal citation, and DOI.
}

theories. On the other hand, experimental observations of cell swimming suggest direct rotational symmetry breaking of the actomyosin concentration delineating the cell cortex [3,4], implying that liquid-crystalline order is not a prerequisite for self-propulsion in cells.

In this Rapid Communication, we present a field theory of self-propelling active droplets, such as cells, in the absence of liquid-crystalline order. Our theory is given in terms of two scalar fields $\phi$ and $\psi$, which follow symmetry arguments of the Landau theory [13], coupled by the active stress in the momentum conservation equation. The $\phi$ field delineates the interior/exterior of the droplet. The $\psi$ field is an auxiliary field which is related to a conserved scalar describing the local amount of active material $(\rho)$. It is defined via $\rho=\phi-\psi$. The arrangement of interest is where a cortex of nonzero $\rho$ [see the red region in Fig. 1(b)] resides in the outer part of an otherwise passive droplet with constant overall mass density. This arrangement corresponds to a droplet with $\psi>0$ [see the blue core in Fig. 1(b)] superimposed on a larger droplet of $\phi>0$. The difference of the two radii is then the thickness of a cortex surrounding the droplet, rendered contractile by, e.g., the action of myosin motors.

Self-propulsion of the active droplet emerges generically by the spontaneous breakdown of rotational symmetry in the $\rho$ field. Once this is broken, the droplet moves with a speed that increases linearly with the activity strength and decreases linearly with the droplet area fraction within a periodic domain. (We rationalize both scalings analytically.) We then extend our theory to study an array of active droplets and identify an active-Crowley-like instability.

Model. First, with two conserved scalar fields $\phi(r, t)$ and $\psi(\boldsymbol{r}, t)$, we choose the free-energy functional $\mathcal{F}[\phi, \psi]$ as

$$
\begin{aligned}
\mathcal{F}[\phi, \psi]= & \int\left(\frac{a}{2} \phi^{2}+\frac{b}{4} \phi^{4}+\frac{a^{\prime}}{2} \psi^{2}+\frac{b^{\prime}}{4} \psi^{4}-\beta \phi \psi\right. \\
& \left.+\frac{\kappa}{2}|\nabla \phi|^{2}+\frac{\kappa^{\prime}}{2}|\nabla \psi|^{2}\right) d \boldsymbol{r},
\end{aligned}
$$

where $a=-b<0, a^{\prime}=-b^{\prime}<0$, and $\kappa, \kappa^{\prime}, \beta>0$. Although we choose $a=a^{\prime}$ and $b=b^{\prime}$, our results are robust against 


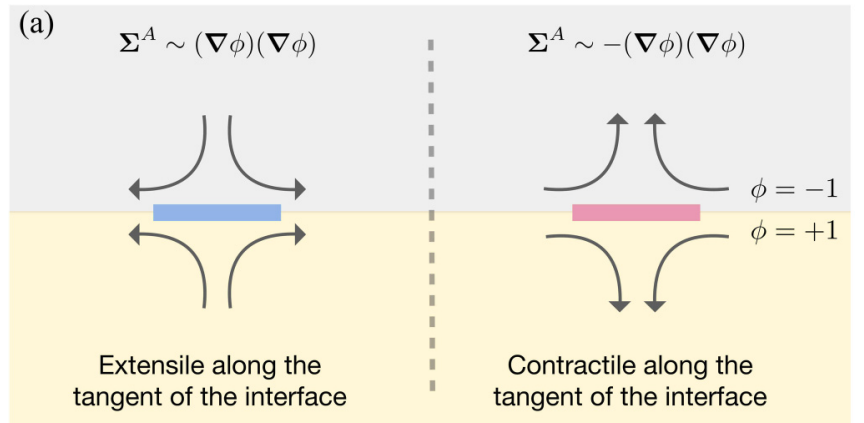

(b)

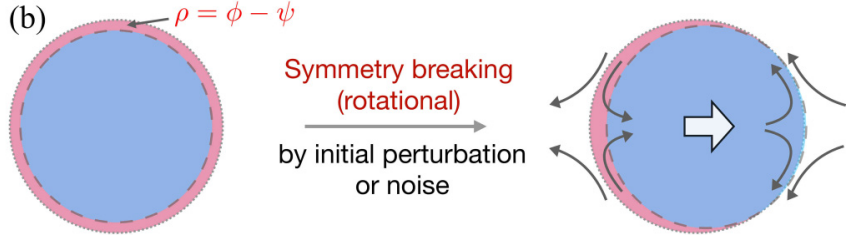

FIG. 1. (a) Flow driven by an active stress $\boldsymbol{\Sigma}^{A}$ on the interface of the droplet phase field $\phi$. A positive (negative) $\boldsymbol{\Sigma}^{A}$ implies an extensile (contractile) — shown by a blue (red) patch-fluid flow tangential to the $\phi$ interface. (b) A cell is represented by a $\phi$ droplet, while the concentration of actomyosin in the cell cortex is given by $\phi-\psi$ (red ring). Under a small random initial perturbation, the $\psi>0$ domain is displaced to the front (right), creating excess contractile stress at the back. This leads to an active flow which locks the $\psi$ droplet in the front, causing sustained self-propulsion along a direction selected by spontaneous symmetry breaking.

changing these parameters as long as the free energy admits the solution for a stable droplet. Connections of these freeenergy parameters to physical parameters-surface tension, interfacial width, thickness of cortex, etc.- - are given in Table 1 of the Supplemental Material (SM) [14].

Equation (1) is adopted as the simplest way to stabilize two concentric phase-field domains (in $\phi$ and $\psi$ ) of unequal size. On identification of $\rho=\phi-\psi$, this becomes a droplet surrounded by a cortex, as required. Parameters are chosen so that each phase field approaches \pm 1 in the interior/exterior bulk phases. The $\beta>0$ term is an energetic coupling which favors maximizing the overlap of $\psi$ and $\phi$ fields. Since both fields are conserved, the volumes of these $\phi, \psi$ droplets (equivalently, the $\phi$ droplet and its $\phi-\psi$ cortex) are separately constant in time. We choose the initial volume of the $\phi$ droplet to be bigger than of the $\psi$ droplet, and thus the $\psi$ droplet resides within the $\phi$ droplet, giving a cortex in between [see Fig. 1(b)]. The $\phi$ droplet then has interfacial tension $\gamma_{0} \simeq \sqrt{-8 \kappa a^{3} / 9 b^{2}}$ [15], with similar expressions for the $\psi$ droplet. These tensions govern respectively the cortex/exterior and cortex/interior interfaces. Note that the $\beta$ term also renormalizes the interfacial tension $\gamma_{0}$; however, we choose $\beta / a=\beta / a^{\prime}=0.01$, so that this difference is not appreciable.

The only active term in our model is a contractile stress, which lives, for simplicity, at the outer interface where $\phi$ passes through zero. This active stress is, however, modulated by the local concentration of cortical material which (in some units) is $\rho(\boldsymbol{r}, t)=\phi-\psi \simeq-\psi>0$ [see Fig. 2(a)]. If rotational symmetry is maintained, the cortex is concentric with the droplet and the active stress is likewise symmetric
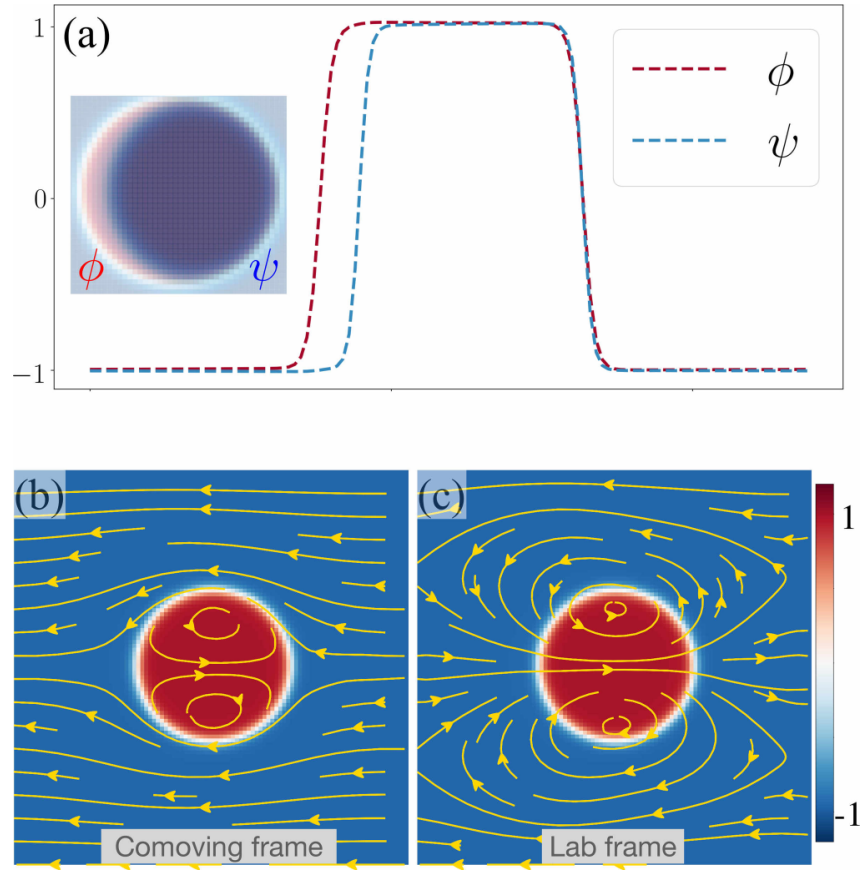

FIG. 2. (a) Inset: A snapshot of an active droplet steadily moving in the $x$ direction. Main: Corresponding plots of $\phi$ and $\psi$ as a function of $x$ at the cross section passing through the center of the droplet. (b) and (c) show the streamlines of the fluid flow of the same active droplet in (b) the comoving frame and (c) laboratory frame. The streamlines are superimposed on the pseudocolor plot of the $\phi$ field.

[Fig. 1(b), left]. In the broken symmetry state, with more cortical material at the rear of the droplet, the active stress is larger there, creating a fluid flow that sustains the broken symmetry, by sweeping the cortex of actomyosin towards the rear so that $-\psi$ is larger there than at the front [Fig. 1(b), right].

This flow is governed by the hydrodynamic velocity $\boldsymbol{v}(\boldsymbol{r}, t)$, which describes the average velocity of the cellular materials plus the solvent. The conserved dynamics of $\phi$ and $\psi$ is then as follows,

$$
\begin{aligned}
\frac{\partial \phi}{\partial t}+\nabla \cdot\left(\phi v-M^{\phi} \nabla \frac{\delta \mathcal{F}}{\delta \phi}\right) & =0, \\
\frac{\partial \psi}{\partial t}+\nabla \cdot\left(\psi v-M^{\psi} \nabla \frac{\delta \mathcal{F}}{\delta \psi}\right) & =0,
\end{aligned}
$$

such that the total $\int \phi d \boldsymbol{r}$ and $\int \psi d \boldsymbol{r}$ are constant in time. The first term inside the parentheses describes advection of $\phi$ and $\psi$ by the fluid velocity $\boldsymbol{v}$. The second term describes diffusion of $\phi$ and $\psi$ along the negative gradient of the chemical potential $\delta \mathcal{F} / \delta \phi$ and $\delta \mathcal{F} / \delta \psi$, respectively. $M^{\phi, \psi}>0$ are the mobilities for each field.

In the limit of low Reynolds number, which is appropriate for subcellular materials, the fluid flow $\boldsymbol{v}(\boldsymbol{r}, t)$ is obtained from solving the Stokes equation,

$$
\nabla \cdot\left(\sigma+\Sigma^{E}+\Sigma^{A}\right)=\mathbf{0},
$$

where $\boldsymbol{\sigma}=-p \boldsymbol{I}+\eta\left[(\boldsymbol{\nabla} \boldsymbol{v})+(\boldsymbol{\nabla} \boldsymbol{v})^{T}\right]$ is the Cauchy stress tensor in a fluid of viscosity $\eta, \boldsymbol{I}$ is the identity matrix, and $p$ is the 
isotropic pressure, which enforces the incompressibility condition $\boldsymbol{\nabla} \cdot \boldsymbol{v}=\mathbf{0} . \boldsymbol{\Sigma}^{E}$ in (3) is the equilibrium interfacial stress, which is derived from the free-energy functional (1) [15],

$$
\Sigma^{E}=-\kappa(\nabla \phi)(\nabla \phi)-\kappa^{\prime}(\nabla \psi)(\nabla \psi) .
$$

Physically, $\boldsymbol{\Sigma}^{E}$ is the elastic response to a deformation in the interface of the $\phi$ and $\psi$ droplet.

$\boldsymbol{\Sigma}^{A}$ in (3) is the active stress, which drives the system out of equilibrium. The form of $\boldsymbol{\Sigma}^{A}$ is adapted from the active model $\mathrm{H}[16,17]$,

$$
\Sigma^{A}=\alpha \psi(\nabla \phi)(\nabla \phi),
$$

where $\alpha \geqslant 0$ is a cortical contractile activity parameter, such that the equilibrium limit is recovered when $\alpha \rightarrow 0$. From (5), the activity is always localized at the interface of the $\phi$ droplet. This differs from other models of active fluid droplets such as active nematics $[6,11,18,19]$, where the active stress affects the bulk of the interior. The physical significance of $\boldsymbol{\Sigma}^{A}$ is illustrated in Fig. 1(a). Consider a patch of active region on an interface separating $\phi=+1$ and $\phi=-1$ regions [see Fig. 1(a)]. If $\alpha \psi>0$ so that $\Sigma^{A} \propto(\nabla \phi)(\nabla \phi)$, the active stress creates an extensile fluid flow in the tangential direction of the interface. On the other hand, if $\alpha \psi<0$, as will hold here, $\boldsymbol{\Sigma}^{A} \propto-(\nabla \phi)(\nabla \phi)$, and the active stress creates a contractile flow tangential to the interface, corresponding to actomyosin contractility $\alpha>0$ in the cell cortex. (Recall that the density of this cortex is $\rho \sim-\psi>0$ at the outer droplet interface.) Note that in the literature on active phase separation $[16,17]$, contractile and extensile are defined with respect to the microswimmer orientation, normal to the interface. The opposite convention, chosen here, refers to the tangential cortex layer and is used in the cellular literature [3,4].

Our numerical system consists of a two-dimensional (2D) square box with linear size $L$ and periodic boundary conditions. We initialize a $\phi$ droplet with radius $R$ at the center of the box, and similarly a $\psi$ droplet with a smaller radius $0.9 R$; see Fig. 1(b), left. We then solve Eqs. (2a)-(3) numerically using a pseudospectral method, as detailed in the SM [14].

Mechanism of self-propulsion. Now we will illustrate the mechanism for self-propulsion. We fix the activity $\alpha$ to be finite and positive. First, let us consider what happens when both $\phi$ and $\psi$ droplets are concentric as shown in Fig. 1(b), left. At the interface of the $\phi$ droplet, the value of $\psi$ is negative and the same everywhere along the interface $(\psi \simeq-1)$. Thus, the active stress $\Sigma^{A}(5)$ is contractile and its magnitude is the same everywhere along the interface. This represents an isotropic distribution of active cortex material, and thus, by symmetry, we should not expect to see any motion.

Now imagine that we give a small displacement (which can come either from thermal fluctuations or an initial perturbation-we consider the latter case here) to the $\psi$ droplet as shown in Fig. 1(b), right. The essence of the symmetry breaking mechanism can be understood by considering a small initial displacement of the smaller droplet $\psi$ such that the interface of the $\phi$ droplet touches that of the $\psi$ droplet at one point (found below to be the "front" of the droplet when in motion) so that the $\rho$ cortex vanishes in thickness at this point. Therefore, at the front, the active stress $\boldsymbol{\Sigma}^{A}(5)$ is approximately zero, whereas at the back, the active stress is finite and contractile. This excess contractile stress at the back pulls the fluid from the front to the back along the interface of $\phi$, as indicated by black arrows in Fig. 1(b), right. This translates into persistent motion. Figure 2(a) shows the values of $\phi$ and $\psi$ measured at the cross section, which supports this mechanism. Physically, we have an accumulation of actomyosin at the back of the cell which creates excess contractility at the rear cell cortex. This is consistent with experiments on cellular swimming motility in the absence of any boundary on which to crawl $[4,20]$.

Figures 2(b) and 2(c) show the steady state fluid velocity from the full hydrodynamic simulations in the comoving [Fig. 2(b)] and laboratory frame [Fig. 2(c)]. This fluid flow is as schematically shown in Fig. 1(b), right. Inside the $\phi$ droplet, the differential active stress at the interface generates a pair of counter-rotating vortices, which then squash the $\psi$ droplet further to the front, giving a positive feedback.

Incidentally, this type of fluid flow is generic to all neutral (pure quadrupolar flow [21]) squirmer droplets, whose motion is typically driven by Marangoni flow along the interface [22-24]. From Figs. 1(b) and 2(a), in the front hemisphere, $\psi=\psi_{f} \simeq 0$, whereas in the back hemisphere, $\psi=\psi_{b} \simeq-1$. The active stress $\boldsymbol{\Sigma}^{A}$ renormalizes the surface tension into an effective surface tension, $\gamma_{0} \rightarrow \gamma=\gamma_{0}(1-$ $\alpha \psi / \kappa)[16,17]$, where $\gamma_{0}=\sqrt{-8 \kappa a^{3} / 9 b^{2}}[15]$ is the surface tension without activity, $\alpha=0$. This has the effect of a net change in surface tension between the front and rear of the $\phi$ droplet, which is $\Delta \gamma=\alpha \Delta \psi \gamma_{0} / \kappa$, where $\Delta \psi=\psi_{f}-\psi_{b}$. Thus, we have a Marangoni flow from low effective surface tension (front) to high effective surface tension (back) as shown in Fig. 2(b), while Fig. 2(c) contains the same flow in the laboratory frame. The corresponding theoretical flow around an active droplet, in an infinite 2D domain, is given in the first figure of the SM [14].

Self-propulsion speed $U$. Having described the mechanism, we now obtain an analytical expression for $U$ using appropriate boundary conditions corresponding to our model. In simulations, the interface is diffused, and thus there is no discontinuity in the fluid velocities inside $(\tilde{\boldsymbol{v}})$ and outside $(\boldsymbol{v})$ the droplet. In analytical calculations, the interface is sharp, and we solve the Stokes equation both inside and outside of the droplet using the following boundary conditions,

$$
\begin{aligned}
v_{r}(r=R) & =\tilde{v}_{r}(r=R)=0, \\
v_{\theta}(r=R) & =\tilde{v}_{\theta}(r=R), \\
(\boldsymbol{\sigma}-\tilde{\boldsymbol{\sigma}}) \cdot \hat{\boldsymbol{r}} & =\gamma(\nabla \cdot \hat{\boldsymbol{r}}) \hat{\boldsymbol{r}}-\nabla \gamma, \quad \text { at } r=R .
\end{aligned}
$$

The above equations represent no flux (6a), continuity of tangential slip velocity (6b) at the interface, and the fact that the discontinuity of the Cauchy stress $\sigma$ at the interface is related to the surface tension $\gamma(\theta)$ of the droplet, via $(6 \mathrm{c})[25,26]$. The speed $U_{0}$ of the droplet in an infinite 2D domain (see SM [14]) is then

$$
U_{0}=\frac{\Delta \gamma}{16 \eta}
$$

As described previously, $\Delta \gamma=\alpha \Delta \psi \gamma_{0} / \kappa$, and thus the speed increases linearly with contractile activity $\alpha$ and actomyosin concentration in the cortex $\rho$. Using the above formula and the parameters of Fig. 3, the theoretically predicted speed is $U_{0}=0.49 \alpha$. This is in excellent agreement with the 

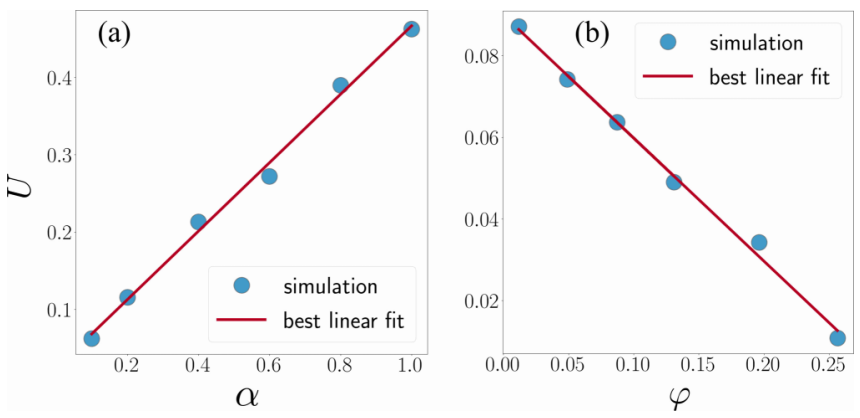

FIG. 3. (a) The self-propulsion speed of the droplet $U$ increases linearly with the activity parameter $\alpha$ at a fixed area fraction $\varphi=$ $\pi R^{2} / L^{2}=0.049$. (b) The self-propulsion speed of the droplet $U$ decreases linearly with the area fraction $\varphi$ for a fixed $\alpha=0.2$. From the best linear fit of the simulation data we obtain $U=0.44 \alpha(1-3.4 \varphi)$, while the theoretical estimate of Eq. (7) is $U=0.49 \alpha$ in the limit $\varphi \rightarrow 0$.

numerical estimate of $U_{0}=0.44 \alpha$ obtained from a best fit of the simulation data of Fig. 3. It should be noted that the speed does not depend on $R$, which is consistent with the literature on transport by interfacial forces [25,27]. We ignore any deformations of the droplet in our calculations. A more detailed analysis studying the role of deformation will be pursued in a future work.

To account for the periodic boundary conditions used in simulations, we need to sum the flow due to periodic images of the droplet, which lie on a $2 \mathrm{D}$ square lattice. The expression for the speed $U$ is then $U=U_{0}(1-\varphi)$ [14]. The best fit of numerical data in Fig. 3 gives $U=0.44 \alpha(1-3.4 \varphi)$. Although our analytical results give the linear scalings for the self-propulsion speed, as a function of activity parameter $\alpha$ and area fraction $\varphi$, seen in the simulations, this belies the true complexity of the problem which exhibits linear scaling with volume fraction far beyond any perturbative regime (and indeed with a different coefficient). A more detailed analysis of the above will be pursed in a future work.

Crowley-like instability of an array of active droplets. The model and simulations above can be easily extended to the case of multiple droplets. This is operationally done by generalizing the free-energy functional of (1) for $N$ active droplets as

$$
\begin{aligned}
\mathcal{F}[\boldsymbol{\phi}, \boldsymbol{\psi}]= & \int \sum_{i=1}^{N}\left(\frac{a}{2} \phi_{i}^{2}+\frac{b}{4} \phi_{i}^{4}+\frac{a^{\prime}}{2} \psi_{i}^{2}+\frac{b^{\prime}}{4} \psi_{i}^{4}-\beta \phi_{i} \psi_{i}\right. \\
& \left.+\frac{\kappa}{2}\left(\nabla \phi_{i}\right)^{2}+\frac{\kappa^{\prime}}{2}\left(\nabla \psi_{i}\right)^{2}+\sum_{j \neq i} \beta^{\prime} \phi_{i} \phi_{j}\right) d \boldsymbol{r} .
\end{aligned}
$$

Here, the term proportional to $\beta^{\prime}>0$ effectively leads to repulsion between the droplets $\phi_{i}$ and $\phi_{j}$ [28], and thus precludes any overlap. The dynamics for each $\phi_{i}, \psi_{i}$, and $v$ is the same as in (2a)-(3) and the equilibrium interfacial stress $\boldsymbol{\Sigma}^{E}$ and active stress $\boldsymbol{\Sigma}^{A}$ are now the sum of each contribution from $\phi_{i}$ and $\psi_{i}$ given in (4) and (5).

We use the above to study the instability in a linear array of three droplets as shown in Fig. 4. We see an active-Crowley-
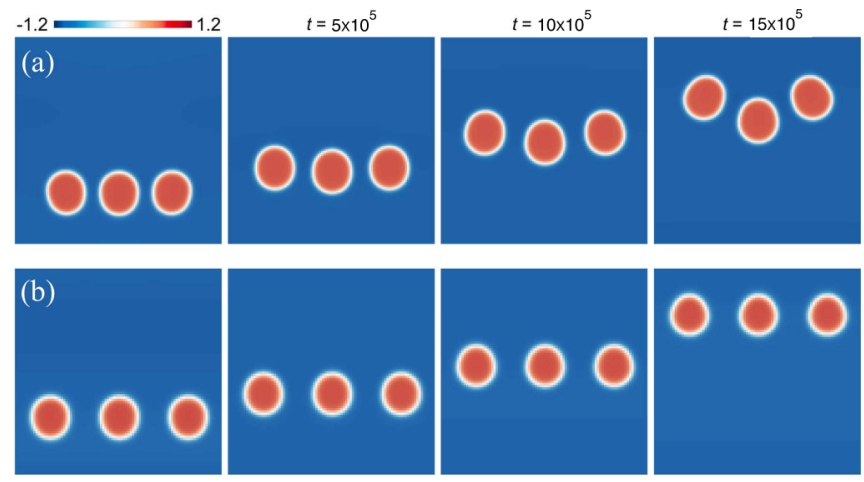

FIG. 4. (a) Active-Crowley-like instability: A linear array of active droplets is unstable. This instability is opposite to that in an array of sedimenting particles. (b) There is no instability if there is no well-defined central particle.

like instability when there is a well-defined central particle. Unlike in the case of the sedimentation [29], here the central particle lags. This can be understood from the fluid flow in Fig. 2(c), which has the effect of the central particle being pushed backwards by the neighboring particles. On the other hand, if the particles are equally separated as in Fig. 4(b), there is no instability as there is no well-defined central particle, when taking into account the periodic boundary condition used.

Conclusion. We have presented a minimal (and scalable) hydrodynamic model of active droplets without any liquidcrystalline order parameter [7,11], nor do we require explicit chemical reactions in the form of source and sink terms [30-33]. The self-propulsion of droplets in our model relies on the fact that excess contractility in the back of the droplet gives rise to a finite effective surface tension gradient, $\Delta \gamma \neq 0$. Thus, although motivated by a simple description of swimming cells, our model can also capture the self-propulsion due to Marangoni stresses on the surface of active emulsion droplets [22-24,34-36]. The effective tension in our case is not a prescribed quantity, but is instead a consequence of the minimal form of the active stress $\boldsymbol{\Sigma}^{A}$ given in Eq. (5). Our theory might possibly be extended to address a passive liquid crystal inside an active scalar droplet, to mimic experimental systems of Refs. [22,34], or, in the chiral case, the helical trajectories seen in experiments of Ref. [37].

We also showed the feasibility of our model for the study of many droplets by studying an active-Crowley-like instability in a linear array of active droplets. A more detailed study of an active droplet suspension using our theory and its comparison to the particulate theories [38] of active matter will be presented in future work.

Acknowledgments. R.S. is funded by a Royal SocietySERB Newton International Fellowship. M.E.C. is funded by the Royal Society. Numerical work was performed on the Fawcett HPC system at the Centre for Mathematical Sciences. Work funded in part by the European Research Council under the Horizon 2020 Programme, ERC Grant Agreement No. 740269. 
[1] M. C. Marchetti, J. F. Joanny, S. Ramaswamy, T. B. Liverpool, J. Prost, M. Rao, and R. A. Simha, Hydrodynamics of soft active matter, Rev. Mod. Phys. 85, 1143 (2013).

[2] T. M. Svitkina, A. B. Verkhovsky, K. M. McQuade, and G. G. Borisy, Analysis of the actin-myosin II system in fish epidermal keratocytes: Mechanism of cell body translocation, J. Cell Biol. 139, 397 (1997).

[3] R. J. Hawkins, R. Poincloux, O. Bénichou, M. Piel, P. Chavrier, and R. Voituriez, Spontaneous contractility-mediated cortical flow generates cell migration in three-dimensional environments, Biophys. J. 101, 1041 (2011).

[4] R. Poincloux, O. Collin, F. Lizárraga, M. Romao, M. Debray, M. Piel, and P. Chavrier, Contractility of the cell rear drives invasion of breast tumor cells in 3D Matrigel, Proc. Natl. Acad. Sci. USA 108, 1943 (2011).

[5] T. Sanchez, D. T. N. Chen, S. J. DeCamp, M. Heymann, and Z. Dogic, Spontaneous motion in hierarchically assembled active matter, Nature (London) 491, 431 (2012).

[6] P. Guillamat, Ž. Kos, J. Hardoüin, J. Ignés-Mullol, M. Ravnik, and F. Sagués, Active nematic emulsions, Sci. Adv. 4, eaao1470 (2018).

[7] E. Tjhung, A. Tiribocchi, D. Marenduzzo, and M. E. Cates, A minimal physical model captures the shapes of crawling cells, Nat. Commun. 6, 5420 (2015).

[8] F. Ziebert and I. S Aranson, Computational approaches to substrate-based cell motility, npj Comput. Mater. 2, 16019 (2016).

[9] B. A. Camley and W. J. Rappel, Physical models of collective cell motility: From cell to tissue, J. Phys. D 50, 113002 (2017).

[10] A. Loisy, J. Eggers, and T. Liverpool, How many ways a cell can move: The modes of self-propulsion of an active drop, Soft Matter 16, 3106 (2020).

[11] E. Tjhung, D. Marenduzzo, and M. E. Cates, Spontaneous symmetry breaking in active droplets provides a generic route to motility, Proc. Natl. Acad. Sci. USA 109, 12381 (2012).

[12] Physical Models of Cell Motility, edited by I. S. Aranson (Springer, Berlin, 2016).

[13] P. M. Chaikin and T. C. Lubensky, Principles of Condensed Matter Physics (Cambridge University Press, Cambridge, UK, 2000).

[14] See Supplemental Material at http://link.aps.org/supplemental/ 10.1103/PhysRevResearch.2.032024 for the detailed calculations, numerical method, and details of the supplemental movies.

[15] M. E. Cates and E. Tjhung, Theories of binary fluid mixtures: From phase-separation kinetics to active emulsions, J. Fluid Mech. 836, P1 (2017).

[16] A. Tiribocchi, R. Wittkowski, D. Marenduzzo, and M. E. Cates, Active Model H: Scalar Active Matter in a MomentumConserving Fluid, Phys. Rev. Lett. 115, 188302 (2015).

[17] R. Singh and M. E. Cates, Hydrodynamically Interrupted Droplet Growth in Scalar Active Matter, Phys. Rev. Lett. 123, 148005 (2019).

[18] M. L. Blow, S. P. Thampi, and J. M. Yeomans, Biphasic, Lyotropic, Active Nematics, Phys. Rev. Lett. 113, 248303 (2014).

[19] L. Giomi and A. DeSimone, Spontaneous Division and Motility in Active Nematic Droplets, Phys. Rev. Lett. 112, 147802 (2014).
[20] V. Ruprecht, S. Wieser, A. Callan-Jones, M. Smutny, H. Morita, K. Sako, V. Barone, M. Ritsch-Marte, M. Sixt, R. Voituriez, and C.-P. Heisenberg, Cortical contractility triggers a stochastic switch to fast amoeboid cell motility, Cell 160, 673 (2015).

[21] S. Kim and S. J. Karrila, Microhydrodynamics: Principles and Selected Applications (Butterworth-Heinemann, Boston, 1991).

[22] S. Thutupalli, R. Seemann, and S. Herminghaus, Swarming behavior of simple model squirmers, New J. Phys. 13, 073021 (2011).

[23] C. Jin, C. Krüger, and C. C. Maass, Chemotaxis and autochemotaxis of self-propelling droplet swimmers, Proc. Natl. Acad. Sci. USA 114, 5089 (2017).

[24] A. Izzet, P. G. Moerman, P. Gross, J. Groenewold, A. D. Hollingsworth J. Bibette, and J. Brujic, Tunable Persistent Random Walk in Swimming Droplets, Phys. Rev. X 10, 021035 (2020).

[25] M. Schmitt and H. Stark, Marangoni flow at droplet interfaces: Three-dimensional solution and applications, Phys. Fluids 28 , 012106 (2016).

[26] M. D. Leven and J. Newman, The effect of surfactant on the terminal and interfacial velocities of a bubble or drop, AIChE J. 22, 695 (1976).

[27] J. L. Anderson, Colloid transport by interfacial forces, Annu. Rev. Fluid Mech. 21, 61 (1989).

[28] M. Foglino, A. N. Morozov, O. Henrich, and D. Marenduzzo, Flow of Deformable Droplets: Discontinuous Shear Thinning and Velocity Oscillations, Phys. Rev. Lett. 119, 208002 (2017).

[29] S. Ramaswamy, Issues in the statistical mechanics of steady sedimentation, Adv. Phys. 50, 297 (2001).

[30] S. Yabunaka, T. Ohta, and N. Yoshinaga, Self-propelled motion of a fluid droplet under chemical reaction, J. Chem. Phys. 136, 074904 (2012).

[31] F. Fadda, G. Gonnella, A. Lamura, and A. Tiribocchi, Lattice Boltzmann study of chemically-driven self-propelled droplets, Eur. Phys. J. E 40, 112 (2017).

[32] M. Morozov and S. Michelin, Nonlinear dynamics of a chemically-active drop: From steady to chaotic self-propulsion, J. Chem. Phys. 150, 044110 (2019).

[33] I. Lavi, N. Meunier, R. Voituriez, and J. Casademunt, Motility and morphodynamics of confined cells, Phys. Rev. E 101, 022404 (2020).

[34] S. Thutupalli, D. Geyer, R. Singh, R. Adhikari, and H. A. Stone, Flow-induced phase separation of active particles is controlled by boundary conditions, Proc. Natl. Acad. Sci. USA 115, 5403 (2018).

[35] M. Morozov and S. Michelin, Self-propulsion near the onset of Marangoni instability of deformable active droplets, J. Fluid Mech. 860, 711 (2019).

[36] D. Lohse and X. Zhang, Physicochemical hydrodynamics of droplets out of equilibrium: A perspective review, arXiv:2005.03782.

[37] T. Yamamoto and M. Sano, Chirality-induced helical selfpropulsion of cholesteric liquid crystal droplets, Soft Matter 13, 3328 (2017).

[38] M. R. Shaebani, A. Wysocki, R. G. Winkler, G. Gompper, and H. Rieger, Computational models for active matter, Nat. Rev. Phys. 2, 181 (2020). 